ARTIGO ORIGINAL ORIGINAL ARTICLE

\section{Os efeitos do memorando de entendimento nas Unidades Locais de Saúde: o caso da Unidade Local de Saúde de Castelo Branco}

\author{
The effects of the memorandum of understanding in the Local \\ Health Units: the case of the Local Health Unit of Castelo Branco
}

Sérgio Farias ${ }^{1}$, Alexandre Morais Nunes²

DOI: 10.21115/JBES.v11.n2.p161-9

\author{
Palavras-chave: \\ austeridade, gestão \\ hospitalar, Análise Envoltória \\ de Dados, eficiência
}

\section{Keywords:}

austerity, hospital management, Data Envelopment Analysis, efficiency

\begin{abstract}
RESUMO
Objetivo: Verificar se o Memorando de Entendimento sobre Condicionalismos Específicos de Política Económica (MECPE), imposto em Portugal em período de crise pelo Fundo Monetário Internacional, Banco Central Europeu e Comissão Europeia, gerou melhoria nos resultados de eficiência no sector da Saúde. Métodos: Foi realizado um estudo de caso para avaliação dos resultados de eficiência nos departamentos clínicos da Unidade Local de Saúde de Castelo Branco (ULSCB), localizada em uma das áreas economicamente mais desfavorecidas Recorreu-se à técnica da Análise Envoltória de Dados, levando em consideração como insumos os custos e como resultados a produção registada de cada departamento clínico em três períodos de tempo antes (2002-2010), durante (2011-2014) e após a austeridade (2015-2017). Os dados referentes ao custo e produção foram fornecidos pelo Gabinete de Apoio à Gestão e pelo Gabinete de Contabilidade do hospital. Em complemento da análise quantitativa, utilizou-se o método qualitativo de análise do conteúdo das entrevistas semiestruturadas, aos diretores e administradores do hospital, que permitiu avaliar a informação que detém e que é resultado do seu treinamento e sua experiência. Resultados: Durante o período de austeridade e execução do MECPE, a unidade hospitalar estudada obteve eficiência técnica média de 0,84, que significou um aumento de 16\% em relação ao período anterior. Em particular, apenas um departamento clínico (neurologia) não registou essa melhoria em sua eficiência técnica. Na análise qualitativa do estudo, a maioria dos entrevistados sentiu que a austeridade teve impacto negativo na eficiência de seus departamentos e de seu hospital, o que não se confirma pelos resultados obtidos. Conclusões: O MECPE não apenas aumentou a eficiência técnica, mas também incentivou medidas inovadoras para aumentar os ganhos nos anos subsequentes.
\end{abstract}

Recebido em: 24/05/2019. Aprovado para publicação em: 12/08/2019.

1. Doutorando em Gestão na Universidade da Beira Interior, Covilhã, Portugal.

2. Universidade de Lisboa, Instituto Superior de Ciências Sociais e Políticas (ISCSP-ULisboa), Centro de Administração e Políticas

Públicas (CAPP); professor auxiliar do ISCSP-ULisboa; coordenador adjunto da Escola de Administração e Gestão de Saúde ISCSP-U-

Lisboa, Lisboa, Portugal.

Auxílio: Não houve recebimentos de financiamento ou equipamento.

Autor correspondente: Sérgio Farias. Telefone: 00351 963158647. E-mail: fariassergio1@hotmail.com 
to record this improvement in its technical efficiency. In the qualitative analysis of the study, most interviewees felt that austerity had a negative impact on the efficiency of their departments and their hospital, which is not confirmed by the results obtained. Conclusions: The Memorandum of Understanding not only increased technical efficiency but also promoted innovative measures that allowed for increased gains in subsequent years.

\section{Introdução}

Em 2008, nos Estados Unidos da América (EUA), uma crise econômica conhecida como a crise do subprime foi desencadeada pela política monetária do Federal Reserve Bank, que estabeleceu taxas de juros muito baixas. Isso resultou em um aumento da oferta de crédito, nomeadamente a clientes de risco que anteriormente não tinham critérios para aprovação. O efeito de contágio fez com que a crise, num processo global, rápidamente se disseminasse para outros países (Cechin \& Montoya, 2017).

Devido à globalização da crise econômica dos EUA, a União Europeia foi contagiada, devido a um conjunto de fatores, nomeadamente: i) a valorização do euro em relação ao dólar; ii) o papel limitado do Banco Central Europeu; iii) a descoordenação econômica; iv) a heterogeneidade fiscal; e v) pelo fato de existirem economias de natureza muito diferente dentro da Zona Euro com facilidade de obtenção de crédito (OPSS, 2012).

Portugal, como membro da União Europeia, foi um dos países que menos cresceu economicamente desde a sua integração no Euro, não permitindo, assim, a convergência com as economias desenvolvidas da Zona Euro, ficando, dessa forma, muito vulnerável à crise (OPSS, 2011).

Em 2011, Portugal esteve no centro da crise e foi forçado a procurar um resgate financeiro na Comissão Europeia (UE), Banco Central Europeu e Fundo Monetário Internacional, com os quais assinou o Memorando de Entendimento sobre Condicionalismos Específicos de Política Económica (MECPE). Nesse momento começou a austeridade que durou até 2014; nesse período houve uma contenção e limitação financeira, que repercutiu na sociedade em geral. O governo lançou uma série de medidas destinadas a reduzir os rendimentos laborais, como a redução de vencimentos, abonos e pensões acima de 1.000 euros, e outras medidas para reduzir as despesas na administração pública (Nunes, 2016a). Em Portugal, durante o período de austeridade, o desemprego e o empobrecimento aumentou, o que teve impacto negativo na saúde da população (OPSS, 2016).

No sector da saúde, o sistema de saúde português enfrentou um aumento constante nos gastos com o fornecimento de cuidados de saúde, em decorrência do aumento da população com idade avançada, do crescente número de doenças crônicas e dos avanços tecnológicos e científicos (Farias \& Nunes, 2019). Essa situação conduziu a uma sobrecarga orçamental (ver Figura 1), o MECPE estabeleceu como objectivo geral aumentar a eficiência e a eficácia dos serviços de saúde, de modo a que exista uma optimização dos serviços e controlando as despesas (Portugal, 2011).

Fez parte do objetivo geral gerar uma redução de custos adicionais na área de medicamentos de modo a diminuir os gastos públicos com medicamentos para 1,25\% do produto interno bruto (PIB) até o final de 2012 e cerca de 1\% do PIB em 2013. Além de reduzir os gastos públicos com o medicamento, pretendeu-se gerar economias adicionais nos custos operacionais dos hospitais (Portugal, 2011).

O MECPE para a saúde incidiu nas seguintes áreas:

- Financiamento;

- Definição de preços e comparticipação de medicamentos;

- Prescrição e monitorização da prescrição;

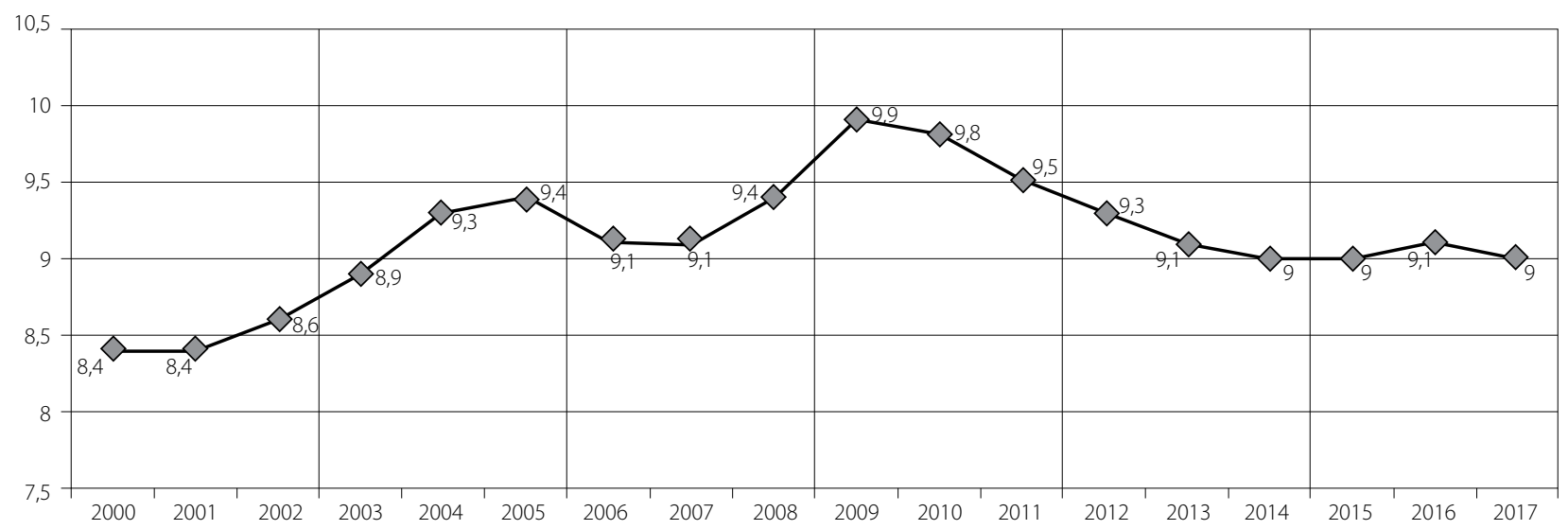

Figura 1. Despesa corrente em cuidados de saúde em \% do PIB, no período de 2000-2015.

Fonte: INE (2018). 
- Sector farmacêutico;

- Compras e aprovisionamento centralizado;

- Cuidados de saúde primários;

- Serviços hospitalares;

- Serviços transversais.

Além do objetivo geral, o MECPE teve como objetivos o controle e a racionalização de gastos e o crescimento da receita no Serviço Nacional de Saúde (SNS) (OPSS, 2012).

Dentro do SNS, os hospitais são uma grande fonte de consumo de recursos, e essa situação pode ser explicada porque os usuários usam essas unidades centrais como uma primeira linha para atenderem as suas necessidades (Nunes, 2017a).

No caso dos serviços hospitalares, o MECPE estabeleceu metas específicas (ver Tabela 1), devido a um rápido aumento nos gastos públicos com a assistência hospitalar, que ultrapassaram 40\% do total de gastos com a assistência em saúde (INE, 2018).

O objeto de estudo deste estudo de caso é a Unidade Local de Saúde de Castelo Branco (ULSCB), que abrange uma população de 108.395 habitantes. A opção por essa unidade deveu-se principalmente ao fato de estar localizada em uma região com recursos limitados e muito envelhecida e também porque, durante o período de austeridade, havia sido penalizada por grandes cortes, como se pode verificar nos valores dos contratos: a) antes da crise, a ULSCB tinha um contrato programa' no valor médio de 74.710.217,35 €; b) durante o período de austeriade (2011-2014), o valor médio dos contrato programa foi de 66.107.837,26 €, ou seja, registou-se um corte superior a 8,6 milhões de euros. Depois da crise (2015-2017), o valor médio do contrato foi de 63.191.264,15 €, o que significou que os ajustes continuaram mesmo depois da crise (ACSS, 2011; ACSS, 2013).

Neste artigo, realizou-se uma análise da eficiência da assistência hospitalar ULSCB e de cada departamento clínico com recurso a técnica da Análise Envoltória de Dados (Data Envelopment Analysis - DEA). Para essa análise, utilizaram-se elementos financeiros e da produção registrada em três períodos: pré-MECPE (2002-2010), durante MECPE (2011-2014) e pós-MECPE (2015-2017). A fim de uma análise mais exaustiva dos resultados obtidos e apronfundar o verdeiro impacto do período de austeridade sentido no hospital pelos respectivos diretores de departamento e administradores, surgiu a questão: "Sentiu alguma limitação na aquisição de recursos (técnicos, humanos) derivada da política de austeridade vivida em todo o sector da saúde entre 2011 e 2014?".
Tabela 1. Objetivos específicos do MECPE para os serviços hospitalares

\begin{tabular}{|c|c|}
\hline Secção & Medida proposta \\
\hline 3.71. & $\begin{array}{l}\text { Liquidação de todos os pagamentos em atraso } \\
\text { (pagamentos devidos a fornecedores nacionais } \\
\text { com atraso superior a } 90 \text { dias). }\end{array}$ \\
\hline 3.72 & $\begin{array}{l}\text { Definir medidas para a redução dos custos } \\
\text { operacionais dos hospitais ( } 200 \text { milhões de euros), } \\
\text { que incluem a redução de cargos dirigentes como } \\
\text { consequência da concentração e racionalização das } \\
\text { unidades. }\end{array}$ \\
\hline 3.73. & $\begin{array}{l}\text { Implementação de um sistema de auditoria e } \\
\text { publicação de normas de orientação clínica. }\end{array}$ \\
\hline 3.74 & $\begin{array}{l}\text { Selecionar, de forma mais transparente, o } \\
\text { presidente e os membros da administração } \\
\text { dos hospitais (por lei, deviam ser pessoas } \\
\text { de reconhecido mérito na saúde, gestão e } \\
\text { administração hospitalar). }\end{array}$ \\
\hline 3.75 & $\begin{array}{l}\text { Desenvolver um sistema de benchmarking que } \\
\text { permita estabelecer uma comparação entre o } \\
\text { desempenho das unidades hospitalares, a ser } \\
\text { publicado, de forma regular, anualmente. }\end{array}$ \\
\hline 3.76. & $\begin{array}{l}\text { Desenvolvimento e interação entre os sistemas } \\
\text { de tecnologias de informação de forma a que a } \\
\text { ACSS tenha em tempo real a informação sobre } \\
\text { as atividades, por forma a publicar relatórios } \\
\text { mensais para os Ministérios da Saúde, das } \\
\text { Finanças e Administração Pública; alcançar a plena } \\
\text { interoperabilidade dos sistemas de informação nos } \\
\text { hospitais. }\end{array}$ \\
\hline 3.77. & $\begin{array}{l}\text { Reorganização e racionalização da rede de } \\
\text { hospitais, através da especialização e concentração } \\
\text { de serviços de urgência, numa gestão conjunta } \\
\text { (Decreto-Lei n 30/2011, de } 2 \text { de março). "Um } \\
\text { plano de acção detalhado será publicado em } 30 \\
\text { de novembro de } 2012 \text { e a sua implementação será } \\
\text { finalizada no primeiro trimestre de } 2013^{\prime \prime} .\end{array}$ \\
\hline 3.78 & $\begin{array}{l}\text { Transferência de serviços hospitalares em } \\
\text { ambulatório para as Unidades de Saúde Familiares } \\
\text { (USF). }\end{array}$ \\
\hline 3.79. & $\begin{array}{l}\text { Realização de um levantamento atual dos médicos } \\
\text { a exercer (por especialidade, idade, região, unidade, } \\
\text { sector público/privado), de forma a identificar as } \\
\text { necessidades atuais e futuras. }\end{array}$ \\
\hline 3.80 & $\begin{array}{l}\text { Publicação de um relatório (até março de 2012) } \\
\text { que contenha os planos de afectação de recursos } \\
\text { humanos até } 2014 \text {. }\end{array}$ \\
\hline 3.81. & $\begin{array}{l}\text { Aplicação de regras para incrementar a mobilidade } \\
\text { de profissionais de saúde dentro e entre cada ARS } \\
\text { (horário flexível, controlo rigoroso das horas de } \\
\text { trabalho e de atividade de cada profissional de } \\
\text { saúde). }\end{array}$ \\
\hline
\end{tabular}

Fonte: Nunes (2016a)

\section{Métodos}

Processo de relacionamento entre financiadores e prestadores assente numa filosofia contratual envolvendo uma ligação entre financiamento atribuído e resultados esperados, considerando metas de produção, acessibilidade e qualidade.
Para concretizar o objetivo de avaliar o impacto da austeridade em um hospital público, o presente estudo recorreu à 
análise da evolução dos escores de eficiência técnica² ${ }^{2}$ dos departamentos clínicos, de imagiologia e patologia clínica, com recurso à técnica da DEA.

A DEA é um método não paramétrico que utiliza um algoritmo matemático para determinar a fronteira de eficiência. Dessa forma, permite avaliar os escores de eficiência de acordo com a razão entre o somatório ponderado dos produtos (outputs) e o somatório ponderado dos insumos (inputs) (Cooper et al., 2007; Hollingsworth et al., 1999).

Na revisão da literatura realizada, verificou-se que a DEA é o método mais comum de análise da efeciência no sector saúde (Cylus et al., 2016). A escolha desse método teve em conta a sua aplicação em estudos nacionais e internacionais relacionados com a temática, como é o caso do estudo de Nunes (2016a), realizado em Portugal sobre as reformas da gestão hospitalar, ou o de Chen et al. (2005), sobre as fontes de ineficiência nos serviços hospitalares dos Estado Unidos da América.

Por meio desse método, consegue-se definir a orientação do estudo, para os insumos (inputs) (representa o valor mínimo dos recursos para cada Decision Making Unit - DMU) e para os resultados (outputs) (maximização da produção, partindo de um conjunto determinado de recursos iniciais) (Cooper et al., 2007).

Os modelos clássicos de DEA podem ser segundo os retornos constantes de escala CRS (Constant Returns to Scale) ou CCR (Charnes, Cooper e Rhodes), ou segundo os retornos variáveis de escala VRS (Variable Returns to Scale) ou BCC (Banker, Charnes e Cooper). No primeiro caso, espera-se uma variação proporcional de produtos a partir da mudança de recursos em todos os níveis de escala. No segundo caso, para certos volumes de recursos gastos, a variação dos produtos perde a proporcionalidade (Lobo \& Lins, 2011). Para avaliar a eficiência total, pode-se utilizar o modelo CRS, o qual permite identificar as unidades eficientes e ineficientes e determinar qual a distância da fronteira de eficiência as unidades efetivas (Azevedo \& Mateus, 2014).

O método da DEA permite avaliar a eficiência técnica das DMUs sem estabelecer valores únicos às ponderações (pesos) para cada variável de input ou output e sem que seja necessário converter todas as variáveis em valores econômicos comparáveis (Pereira \& Mello, 2015).

$\mathrm{Na}$ DEA, verificou-se uma limitação para os casos em que várias DMUs estão no limiar de eficiência, obtendo um escore igual a 1. Assim, de forma a suprimir essa limitação, Andersen \& Petersen (1993) criaram um modelo capaz de medir a supereficiência, de modo a classificar unidades eficientes para modelos radiais (por exemplo, CCR, BCC). (Anderson \& Petersen, 1993; Cooper et al., 2007). Com esse novo modelo, foi possível diferenciar as unidades com escore de eficiência igual a 1 e estabelecer um raking (Cooper et al., 2007; Zhu, 2001).

O conceito de eficiência técnica é definido como a situação em que é impossível que uma unidade de produção produza mais produtos com insumos que está a usar no know-how atual e na tecnologia existente (Cooper et al., 2007).
Segundo a programação fracionária, a ponderação dada às variáveis de inputs $\left(x_{m o}\right)$ é $\left(v_{i}\right)\{i=1, \ldots, m\}$ e de outputs, de $\left(y_{s o}\right)$ é $\left(u_{r}\right)\{r=1, \ldots, s\}$

$$
\max \theta(v, u)=\frac{u_{1} y_{1 o}+u_{2} y_{20+\cdots+u_{s} y_{s o}}}{v_{1} x_{1 o}+v_{2} x_{2 o}+\cdots+v_{m} x_{m o}}
$$

tal que

$$
\begin{aligned}
& \frac{u_{1} y_{1 j}+\cdots+u y_{s j}}{v_{1} x_{1 o}+\cdots+v_{m} x_{m j}} \leq 1 \quad(j=1, \cdots, n) \\
& v_{1}, v_{2}, \cdots, v_{m} \geq 0 \\
& u_{1}, u_{2}, \cdots, u_{s} \geq 0
\end{aligned}
$$

Com a fórmula anterior, obtêm-se infinitas soluções, assim Charnes et al. (1978) propuseram uma resolução de programação linear (dual):

$$
\max \theta(\mu, v)=\mu_{1} y_{10}+\cdots+\mu_{s} y_{s o}
$$

tal que

$$
\begin{aligned}
& v_{1} x_{1 o}+\cdots+v_{m} x_{m o}=1 \\
& \mu_{1} y_{1 j}+\cdots+\mu_{s} y_{s j} \leq v_{1} x_{1 j}+\cdots+v_{m} x_{m j} \quad(j=1, \cdots, n) \\
& v_{1}, v_{2}, \cdots, v_{m} \geq 0 \\
& \mu_{1}, \mu_{2}, \cdots, \mu_{s} \geq 0
\end{aligned}
$$

Para os objetivos propostos, recorreu-se ao modelo de CCR orientado para o output.

$\max (n, \mu) \quad n$

tal que

$$
\begin{aligned}
& x_{o}-X \mu \geq 0 \\
& n y_{o}-Y \mu \leq 0 \\
& \mu \geq 0
\end{aligned}
$$

Confrontados com a homogeneidade dos resultados, optou-se pelo conceito de supereficiência de Andersen \& Petersen (1993).

$$
\theta^{*}=\min \left(\theta, \lambda, s^{-}, s^{+}\right) \theta-\varepsilon e s^{+}
$$

tal que

$$
\begin{aligned}
& \theta x_{o}=\sum_{j=1, \neq 0}^{n} \lambda_{j} x_{j}+s^{-} \\
& y_{o}=\sum_{j=1, \neq 0}^{n} \lambda_{j} y_{j}+s^{+}
\end{aligned}
$$

Para avaliação da eficiência hospitalar, durante os três períodos em estudo, utilizaram-se, como:

- Inputs, os custos totais (diretos e indiretos) efetuados em cada departamento do Hospital Amato Lusitano;

- Outputs, o número de pacientes saídos do internamento, o número total de consultas, o número total de cirurgias e o número total de sessões de hospital de dia. Para o departamento 
de imagiologia os resultados considerados foram radiografias convencionais, tomografia axial computadorizada, mamografia e ultrassonografia; no departamento de patologia clínica, utilizou-se o número de análises realizadas de bioquímica, hematologia, microbiologia e imunologia/virologia.

Os departamentos clínicos do hospital que serviram de amostra ao estudo encontram-se representados na tabela 2.

A fonte para os dados necessários para este estudo foi o Serviço de Gestão Financeira e Gabinete de Apoio à Gestão da ULSCB.

Tabela 2. Departamentos clínicos incluídos no estudo

\begin{tabular}{ll}
\hline Área médica & Cardiologia \\
& Gastroenterologia \\
& Medicina Interna \\
& Nefrologia \\
& Neurologia \\
& Pediatria/Neonatologia \\
& Pneumologia \\
& Psiquiatria (agudos) \\
& Dermatovenerologia \\
\hline Área cirúrgica & Cirurgia Geral \\
& Oftalmologia \\
& Ginecologia/Obstetrícia \\
& Otorrinolaringologia \\
& Ortopedia \\
& Urologia \\
\hline Áreas complementares & Imagiologia \\
& Patologia Clínica \\
\hline
\end{tabular}

Fonte: Elaboração própria.
Outro aspecto do estudo foi o recurso às entrevistas com os sucessivos responsáveis pelos departamentos visados e administradores do hospital, de modo a complementar a análise realizada em termos quantitativos aos efeitos do memorando de entendimento no hospital em questão.

O recurso às entrevistas semiestruturadas possibilitou avaliar a percepção dos entrevistados sobre o problema em estudo, tendo por base a sua formação e experiência.

A análise de conteúdo foi realizada com base em uma categorização prévia, possibilitando, assim, dar sentido a todas as verbalizações coletadas. Essa técnica permite analisar de melhor forma a mensagem transmitida por cada um dos entrevistados (Bardin, 2006).

O objetivo da análise qualitativa, foi trazer mais detalhes para completar os dados, abundância interpretativa, contextualização do meio envolvente e experiências pessoais (Nunes, 2016b).

\section{Resultados}

Nas variáveis utilizadas, realizou-se em primeiro lugar uma análise estatística descritiva, como se pode observar nas tabelas 3 e 4.

A fim de verificar o impacto da MECPE na eficiência técnica do Hospital Amato Lusitano e nos seus departamentos, recorreu-se à DEA, considerando-se uma avaliação global em termos de variação média na eficiência técnica, registada durante os períodos de 2002-2010, 2011-2014 e 2014-2017.

Tabela 3. Valores médios e coeficiente de dispersão das variáveis dos departamentos

\begin{tabular}{|c|c|c|c|c|c|c|c|c|}
\hline Departamentos & $\begin{array}{l}\text { Custo total } \\
\text { (média) }\end{array}$ & $\begin{array}{l}\text { Coeficiente } \\
\text { de } \\
\text { dispersão }\end{array}$ & $\begin{array}{l}\text { No de doentes } \\
\text { saídos do } \\
\text { internamento } \\
\text { (média) }\end{array}$ & $\begin{array}{l}\text { Coeficiente } \\
\text { de } \\
\text { dispersão }\end{array}$ & $\begin{array}{l}\text { No total de } \\
\text { consultas } \\
\text { (média) }\end{array}$ & $\begin{array}{l}\text { Coeficiente } \\
\text { de } \\
\text { dispersão }\end{array}$ & $\begin{array}{l}\text { No total } \\
\text { de } \\
\text { cirurgias } \\
\text { (média) }\end{array}$ & $\begin{array}{c}\text { Coeficiente } \\
\text { de } \\
\text { dispersão }\end{array}$ \\
\hline Cardiologia & 1423927 & 0,26 & 695,56 & 0,16 & 4095,56 & 0,10 & 144,06 & 0,43 \\
\hline Medicina Interna & 3707513 & 0,11 & 1480,19 & 0,10 & 3446,00 & 0,31 & - & - \\
\hline Dermatologia & 214335 & 0,34 & 21,19 & 0,59 & 3019,00 & 0,16 & 75,94 & 0,46 \\
\hline Gastroenterologia & 1528778 & 0,11 & 438,81 & 0,12 & 2795,00 & 0,17 & - & - \\
\hline Nefrologia & 1517383 & 0,14 & 531,88 & 0,22 & 2026,19 & 0,34 & 94,31 & 1,06 \\
\hline Neurologia & 369339 & 0,16 & 141,31 & 0,42 & 1703,19 & 0,19 & - & - \\
\hline Pneumologia & 278943 & 0,45 & 126,88 & 0,43 & 1538,81 & 0,42 & 100,75 & 1,61 \\
\hline Psiquiatria & 1343792 & 0,26 & 275,81 & 0,11 & 5450,50 & 0,34 & - & - \\
\hline Cirurgia Geral & 4671390 & 0,11 & 1936,38 & 0,16 & 7031,19 & 0,22 & 1945,75 & 0,12 \\
\hline Ortopedia & 2361911 & 0,13 & 936,38 & 0,12 & 4672,81 & 0,27 & 901,94 & 0,18 \\
\hline Otorrinolaringologia & 686054 & 0,11 & 251,25 & 0,57 & 4670,69 & 0,17 & 408,50 & 0,28 \\
\hline Urologia & 1427489 & 0,21 & 591,13 & 0,29 & 2954,56 & 0,28 & 358,88 & 0,27 \\
\hline Obstetrícia/Ginecologia & 1894473 & 0,25 & 842,81 & 0,34 & 4325,88 & 0,17 & 447,44 & 0,14 \\
\hline Pediatria/Neonatologia & 1697122 & 0,24 & 816,56 & 0,24 & 3527,94 & 0,12 & 183,06 & 1,12 \\
\hline
\end{tabular}

Fonte: Elaboração própria. 
Tabela 4. Valores médios e coeficiente de dispersão das variáveis dos departamentos das áreas complementares

\begin{tabular}{|c|c|c|c|c|c|c|}
\hline Departamentos & $\begin{array}{l}\text { Custo total } \\
\text { (média) }\end{array}$ & $\begin{array}{l}\text { No total de } \\
\text { análises } \\
\text { bioquímica } \\
\text { (média) }\end{array}$ & $\begin{array}{l}\text { No total de } \\
\text { análises } \\
\text { hematologia } \\
\text { (média) }\end{array}$ & $\begin{array}{l}\text { No total de } \\
\text { análises } \\
\text { microbiologia } \\
\text { (média) }\end{array}$ & $\begin{array}{l}\text { No total de } \\
\text { análises } \\
\text { imunologia/ } \\
\text { virologia } \\
\text { (média) }\end{array}$ & \\
\hline \multirow[t]{3}{*}{ Patologia Clínica } & 1592998 & 451515,80 & 94826,56 & 36100,44 & 6767,13 & \\
\hline & 0,14 & 0,26 & 0,18 & 0,18 & 1,11 & $\begin{array}{l}\text { Coeficiente de } \\
\text { dispersão }\end{array}$ \\
\hline & $\begin{array}{l}\text { Custo total } \\
\text { (média) }\end{array}$ & $\begin{array}{l}\text { No total de RX } \\
\text { (média) }\end{array}$ & $\begin{array}{l}\text { No total de TAC } \\
\text { (média) }\end{array}$ & $\begin{array}{l}\text { No total de } \\
\text { mamografia } \\
\text { (média) }\end{array}$ & $\begin{array}{c}\text { No total de } \\
\text { ecografia (média) }\end{array}$ & \\
\hline \multirow[t]{2}{*}{ Imagiologia } & 1399765 & 51065,94 & 16887,00 & 329,50 & 5894,06 & \\
\hline & 0,27 & 0,07 & 0,35 & 0,41 & 0,38 & $\begin{array}{l}\text { Coeficiente de } \\
\text { dispersão }\end{array}$ \\
\hline
\end{tabular}

Fonte: Elaboração própria.

Tabela 5. Frequência das verbalizações relativamente ao impacto do período de austeridade nos departamentos do Hospital Amato Lusitano

\begin{tabular}{|c|c|c|c|c|}
\hline Categoria & Subcategorias & Unidade de registo & $\mathbf{n}$ & $\%$ \\
\hline \multirow{3}{*}{$\begin{array}{l}\text { Percepção sobre as implicações na } \\
\text { eficiência resultantes das medidas de } \\
\text { contenção de custos impostas pelo } \\
\text { memorando de entendimento }\end{array}$} & \multirow{3}{*}{$\begin{array}{l}\text { Contenções de custos - implicações } \\
\text { em nível da eficiência na prestação } \\
\text { de cuidados }\end{array}$} & Impacto positivo (aumento de eficiência) & 2 & 11,8 \\
\hline & & Impacto negativo (baixou eficiência) & 8 & 47,0 \\
\hline & & $\begin{array}{l}\text { Impacto neutro (sem implicações em } \\
\text { nível da eficiência) }\end{array}$ & 7 & 41,2 \\
\hline
\end{tabular}

Fonte: Elaboração própria.

No período pré-MECPE, 2002-2010, a eficiência técnica média do Hospital Amato Lusitano foi de 0,68. Durante o período 2011-2014, em que Portugal teve de aplicar o MECPE, o Hospital Amato Lusitano obteve uma eficiência técnica média de de 0,84. No período pós-MECPE, 2015-2017, o Hospital Amato Lusitano obteve eficiência técnica média de 0,88.

Os resultados de eficiência técnica média resgistada em cada departamento apresentam-se na figura 2.

$\mathrm{Na}$ análise qualitativa suplementar, obtiveram-se, por parte dos respetivos diretores e administradores, 17 verbalizações e, por meio da técnica da análise de conteúdo, apuraram-se três unidades de registo (vide Tabela 5).

\section{Discussão}

No geral, os resultados são consistentes com um estudo nacional conduzido por Nunes (2016a), que, ao analisar as resoluções da MECPE aplicadas aos hospitais, concluiu que a eficiência técnica foi aumentada pela redução de custos, e não pela redução assistencial.

Com relação ao caso específico dos departamentos da ULSCB, verificou-se que os diferentes departamentos se comportaram de modo desigual no que se refere aos resultados de eficiência técnica, em termos médios, quando comparados o período do MECPE (2011-2014) com o período pré-MECPE (2002-2010):
- Departamentos que melhoraram a sua eficiência: Cardiologia, Nefrologia, Medicina Interna, Psiquiatria, Gastrenterologia, Pneumologia, Cirurgia Geral, Oftalmologia, Ortopedia, Otorrinolaringologia, Dermatovenerologia, Urologia, Obstetrícia/Ginecologia, Pediatria/ Neonatologia, Imagiologia e Patologia Clinica;

- Departamento que agravou os seus resultados de eficiência: Neurologia.

Esses resultados podem ter tido origem em vários fatores como: cortes nos custos dos hospitais com pessoal (nos vencimentos superiores a $1.500 €$ e nas horas extraordinárias), maior pressão para reduzir custos com doentes e reduzir a prescrição de medicamentos e o aumento de recusa de tratamentos inovadores.

Em análise complementar, verificou-se que, após o MECPE (2015-2017), seis departamentos: Pediatria/Neonatologia, Cirurgia Geral, Pneumologia, Gastroenterologia, Cardiologia e Nefrologia agravaram os seus resultados de eficiência em relação ao período do MECPE. Por outro lado, os departamentos de Dermatovenerologia, Psiquiatria, Oftalmologia, Urologia, Obstetrícia/Ginecologia, Imagiologia e Patologia Clínica atingiram um resultado de supereficiência.

Quando comparados os perídos pré-MECPE e pós-MECPE, constatou-se que os serviços de Cardiologia, Neurologia e Cirurgia Geral voltaram a valores de eficiência semelhantes ao 
Cardiologia

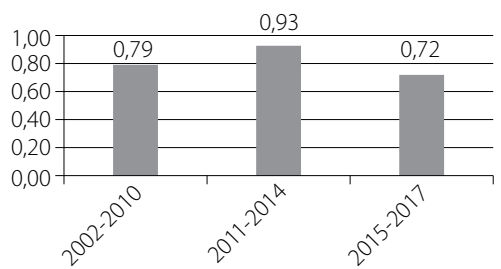

Neurologia

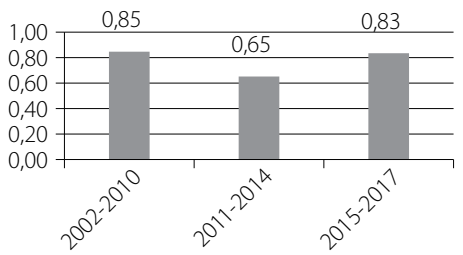

Pneumologia
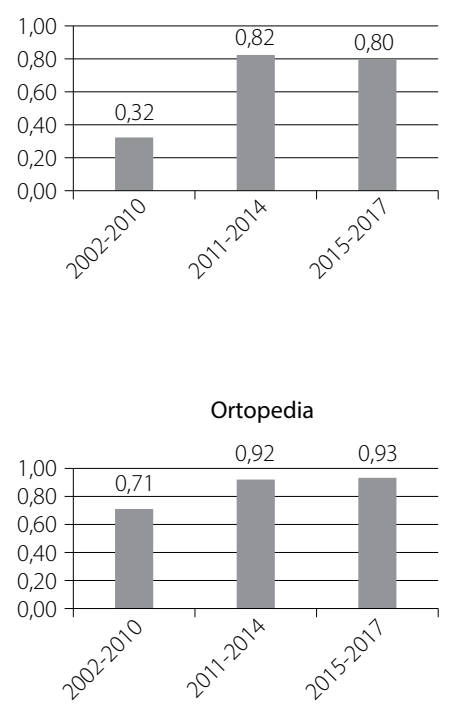

Urologia

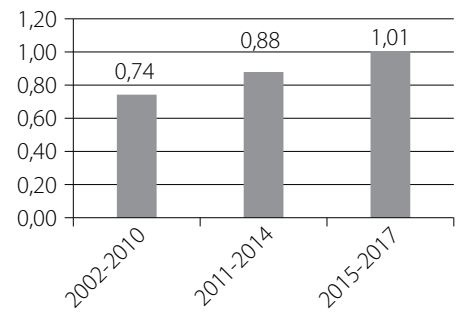

Nefrologia

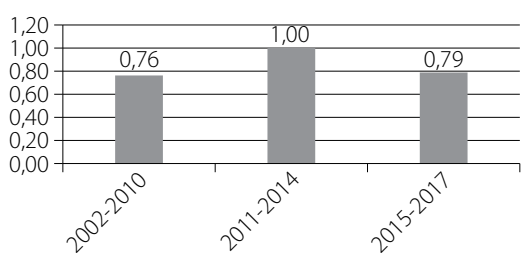

Psiquiatria

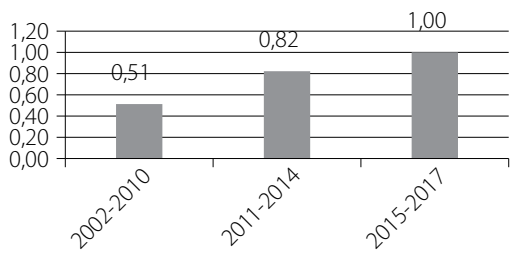

Cirurgia Geral

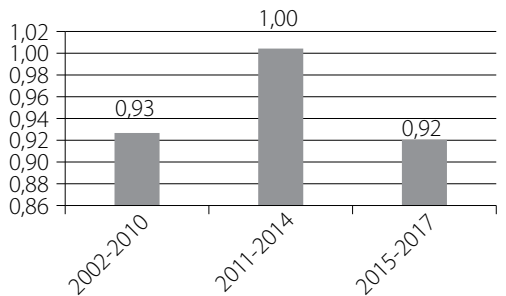

Otorrinolaringologia

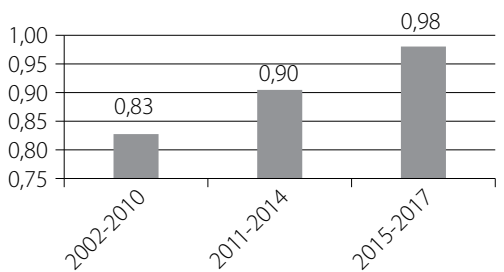

Obstetrícia/Ginecologia

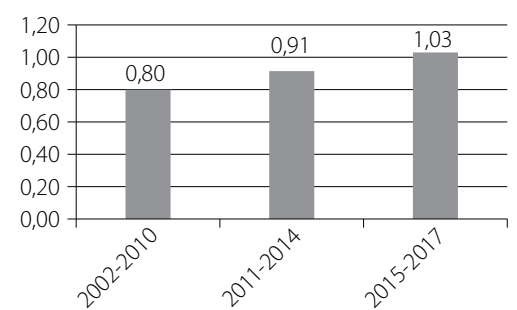

Medicina interna

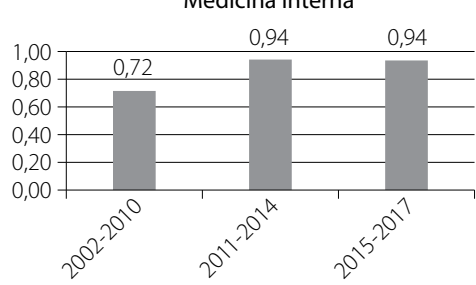

Gastroenterologia

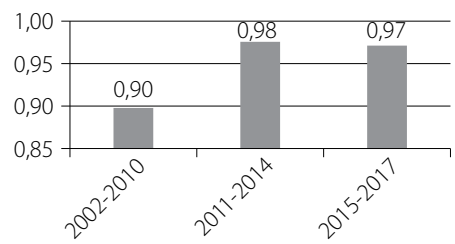

Oftalmologia

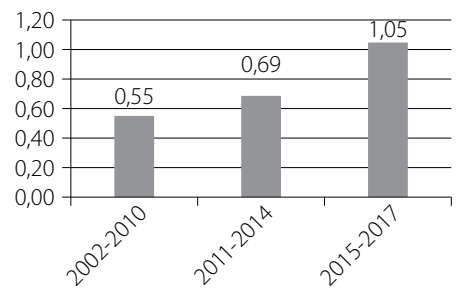

Dematovenerologia

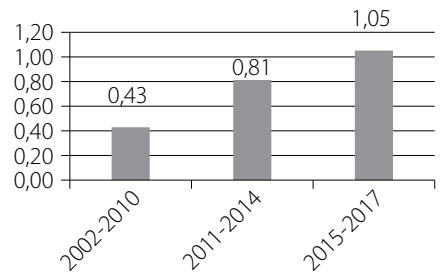

Pediatria/Neonatologia

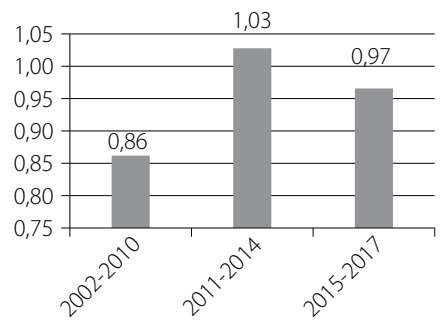

Imagiologia

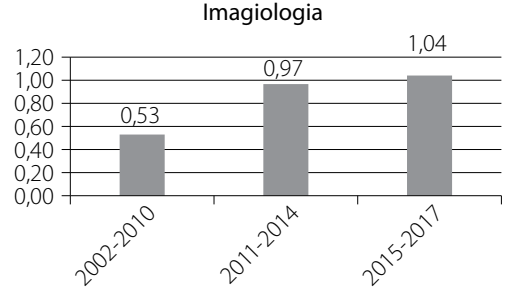

Patologia clínica

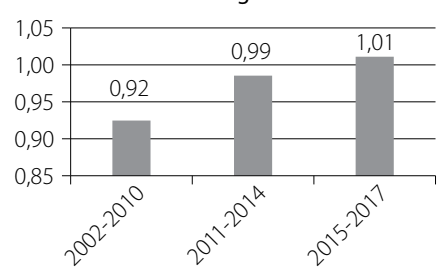

Figura 2. Escores de eficiência técnica média por departamento, antes, durante e após austeridade.

Fonte: Elaboração própria. 
período pré-MECPE. Todos os outros serviços melhoraram os seus escores de eficiência.

No pós-MECPE, a maioria dos departamentos clínicos manteve os escores de eficiência elevados devido à manutenção de alguns compromissos assumidos no MECPE, como os pagamentos em atraso.

$\mathrm{Na}$ análise qualitativa do estudo, em termos de resposta, a maioria dos diretores de departamento e administradores entrevistados (47,0\%) sentiu que o período de austeridade, devido ao MECPE, teve impacto negativo na eficiência nos seus departamentos e hospital. No entanto, $41,2 \%$ dos entrevistados acham que o período de austeridade não teve impacto. E apenas dois entrevistados (11,8\%) referiram que a austeridade teve impacto positivo para a melhoria dos resultados de eficiência hospitalar. Esses resultados são corroborados pelo estudo de Nunes (2016a), que concluiu que os departamentos hospitalares, devido à redução de custos, foram alvo de desinvestimento e de resistência dos profissionais (desmotivados).

É importante mencionar que dois entrevistados relatam que, após a MECPE, permaneceu alguma falta de autonomia na gestão. Essas duas afirmações são consistentes com a realidade, pois, por um lado, grande parte dos termos de referência para a contratualização iniciados em 2013 permaneceu nos atuais contratos. Por outro lado, também existe legislação que limita a autonomia da gestão, por exemplo, os casos de aprovação de despesa segundo o Despacho no 10.220/2014, de 8 de agosto 3 , e a continuidade da vigência da Lei dos Compromissos e dos Pagamentos em Atraso ${ }^{4}$.

\section{Conclusões}

Na análise dos escores de eficiência, concluiu-se que houve aumento na eficiência técnica média durante a austeridade. Embora essa não seja a percepção dos entrevistados, que estão divididos entre um impacto neutro ou negativo, nunca verbalizando uma percepção positiva associada a um incremento de eficiência no período de aplicação das medidas do MECPE.

Relativamente ao efeito pós-MECPE, a maioria dos departamentos registou uma tendência de aumento da eficiência técnica.

Estas conclusões estão em consonância com a bibliografia existente em Portugal, como é o estudo realizado por Nunes (2017b), que concluiu que a austeridade introduzida pelo memorando de entendimento por meio de corte no financiamento atribuído aos hospitais e mantendo o movimento assistencial só poderia resultar numa melhoria da eficiência.

3 Determina que o conselho de administração das entidades do SNS podem autorizar os investimentos que tenham valor inferior a 1.000.000€, desde que as entidades não tenham acumulado pagamentos em atraso no ano anterior ao da realização do investimento.

4 Aprova as regras aplicáveis à assunção de compromissos e aos pagamentos em atraso das entidades públicas.
Farrel (1957) também previu esse resultado, quando em seu trabalho sobre a definição de eficiência falou nos ganhos de eficiência por meio da minimização do insumos. Em Portugal, até ao momento, não se realizaram mais estudos que relacionem o MECPE com avaliação da eficiência com recurso à DEA.

Em conclusão, o MECPE conferiu um aumento da eficiência técnica ao Hospital Amato Lusitano.

\section{Referências bibliográficas}

ACSS - Administração Central do Sistema de Saúde. Contrato Programa 2011. 2011.

ACSS - Administração Central do Sistema de Saúde. Contrato Programa 2013-2015. 2013.

Andersen P, Petersen N. A procedure for ranking efficient units in data envelopment analyses. Management Science. 1993;39(10):1261-4.

Azevedo H, Mateus C. Economias de escala e de diversificação: uma análise da bibliografia no contexto das fusões hospitalares. Rev Port Saúde Pública. 2014;32(1):106-17.

Bardin L. Análise de conteúdo. Lisboa: Edições 70; 2006.

Cechin A, Montoya MA. Origem, causas e impactos da crise financeira de 2008. Teoria e Evidência Econômica. 2017;23(48):150-71.

Charnes A, Cooper W, Rhodes E. Measuring the efficiency of decision making units. Eur J Operat Res. 1978;2:429-44.

Chen A, Hwang Y, Shao B. Measurement and sources of overall and input inefficiencies: Evidences and implications in hospital services. Eur J Operat Res. 2005;161(2):447-68.

Cooper W, Seiford L, Tone K. Data envelopment analysis: a comprehensive text with models, applications, references and DEA-solver software. 2nd ed. Reino Unido: Springer; 2007.

Cylus J, Papanicolas I, Smith PC. Health system efficiency - How to make measurement matter for policy and management. Copenhaga: Organização Mundial da Saúde; 2016.

Farias S, Nunes AM. A integração vertical de cuidados de saúde: resultados de eficiência de um hospital integrado numa unidade local de saúde. Rev Port Gestão Saúde. 2019;25:17-21.

Farrel MJ. The measurement of productive efficiency. J Royal Stat Soc. 1957;120(3):253-81.

Hollingsworth B, Dawson P, Maniadakis N. Efficiency measurement of health care: a review of non-parametric methods and applications. Health Care Manag Sci. 1999;2:161-72.

INE - Instituto Nacional Estatística. 2018. Available from: http://smi.ine.pt/ Conceito.

Lobo MC, Lins ME. Avaliação da eficiência dos serviços de saúde por meio da análise envoltória de dados. Cad Saúde Coletiva. 2011;19(1):93-102.

Nunes AM. Análise da produtividade da política de fusão de unidades hospitalares em Portugal integradas no Serviço Nacional de Saúde. JBES. 2017a;9(1):93-9.

Nunes AM. Crise e volume de internações hospitalares em Portugal. Saúde em Redes. 2017b;3(3):264-72.

Nunes AM. Efeitos da Aplicação do Memorando de Entendimento sobre Condicionalismos Específicos de Política Económica na Prestação de Cuidados Hospitalares. Rev Port Gestão Saúde. 2016a;18:30-3.

Nunes AM. Reformas na gestão hospitalar: análise dos efeitos da empresarialização [tese]. Instituto Superior de Ciências Sociais e Políticas: Lisboa; 2016b.

OPSS - Observatório Português dos Sistemas de Saúde. Crise \& Saúde: um país em sofrimento. Relatório Primavera. 2012. 
OPSS - Observatório Português dos Sistemas de Saúde. Da depressão da crise para a governação prospectiva da saúde. Relatório Primavera. 2011.

OPSS - Observatório Português dos Sistemas de Saúde. Saúde - Procuramse novos caminhos. Relatório Primavera. 2016.

Pereira E, Mello J. Uso da suavização da fronteira na determinação de pesos únicos em modelos DEA CCR. Production. 2015;25(3):585-97.

Portal Unidade Local de Saúde de Castelo Branco (ULSCB). 2019. Available from: http://www.ulscb.min-saude.pt/institucional/orcamentoscontratos-programa-e-planos-de-desempenho/.
Portugal. Memorando de Entendimento sobre as Condicionalidades de Política Económica. Programa de Assistência Financeira UE/FMI/BCE. Request for a Three-Year Arrangement Under the Extended Fund Facility, IMF Country Report No. 11/127, June 2011. Available from: https://www.portugal.gov.pt/media/371372/mou_pt_20110517.pdf. Zhu J. Super-Efficiency and DEA Sensitivity Analysis. Eur J Operat Res. 2001;129(2):443-55. 